\title{
Imaging performance and preoperative differential diagnosis of multiple myopericytoma in the liver: A case report
}

\author{
XI KANG ${ }^{1}$, FANG $\mathrm{LI}^{2}$, HAN GAO ${ }^{1}$ and SHUNXIANG WANG ${ }^{1}$ \\ Departments of ${ }^{1}$ Hepatobiliary Surgery and ${ }^{2}$ Pathology, Hebei Medical University 4th Hospital, \\ Shijiazhuang, Hebei 050011, P.R. China
}

Received September 5, 2019; Accepted April 22, 2020

DOI: $10.3892 / \mathrm{mco} .2020 .2136$

\begin{abstract}
Myopericytoma is a rare type of benign tumor, which commonly affects all four limbs. The occurrence of myopericytoma in the liver is extremely rare. Myopericytoma with a size of $>1 \mathrm{~cm}$ in diameter in the liver has not been previously reported. Due to the limited number of cases, the preoperative diagnosis of liver myopericytoma based on laboratory and imaging examinations is difficult. In the present case report, a patient with multiple myopericytoma in the liver, with a maximum diameter of $4.5 \mathrm{~cm}$ was described, with accompanying computed tomography (CT) and positron emission tomography/CT (PET/CT) imaging profiles. The aim of the present report was to discuss the preoperative differential diagnosis between myopericytoma and other common liver neoplasms, such as intrahepatic cholangiocarcinoma or liver metastasis.
\end{abstract}

\section{Introduction}

Myopericytoma is a rare type of benign tumor, named by Granter in 1998 (1) and classified by the World Health Organization in 2002 (2). This type of tumor is derived from perivascular myoid cells and shares features with both smooth muscle and glomus cells (3). The most commonly affected sites of myopericytoma are the skin and soft tissues of the lower extremities and upper extremities; however, it can also occur in the intracranial space, nose, kidney, and urinary tract (4-8). Reports of myopericytoma occurrence in the liver are extremely rare. Chen and Liang (9) reported a case of myopericytoma,

Correspondence to: Professor Shunxiang Wang, Department of Hepatobiliary Surgery, Hebei Medical University 4th Hospital, 12 Jiankang Road, Shijiazhuang, Hebei 050011, P.R. China

E-mail: gdwangsx@163.com

Abbreviations: CT, computed tomograghy; PET, positron emission tomography; SUV, standardized uptake values; AFP, $\alpha$-fetoprotein; $\mathrm{CEA}$, carcinoembryonic antigen; $\mathrm{CA}$, cancer antigen

Key words: myopericytoma, liver, CT, PET/CT, differential diagnosis which occurred in the area between the liver and stomach in 2017, while Mannan et al (10) reported a case of myopericytoma, which was identified during surgery on segment IV of the liver and was $1.0 \mathrm{~cm}$ in diameter without imaging data in 2016. Due to the limited number of cases reported, the preoperative diagnosis of liver myopericytoma is difficult, and could be mistaken for other types of neoplasms, which occur in the liver, such as intrahepatic cholangiocarcinoma or liver metastasis. In the present report, a case of multiple myopericytoma, which occurred in the liver with a maximum diameter of $4.5 \mathrm{~cm}$ has been described, and the accompanying contrast-enhanced computed tomography (CT) scan and positron emission tomography/CT (PET/CT) scan images are also included to further discuss the imaging features and preoperative differential diagnosis of myopericytoma in the liver.

\section{Case report}

The patient was a 55-year-old female who presented with right upper quadrant tenderness for 2 weeks and was hospitalized at the Hebei Medical University 4th Hospital in December 2018. A non-enhanced CT scan acquired at another local hospital prior to hospitalization showed two low-density lesions adjacent to each other on segments IV and VIII of the liver, suggesting a malignant tumor. Physical examination revealed no palpable abdominal mass and positive right upper quadrant tenderness without rebound tenderness. After being hospitalized, the patient's laboratory blood tests showed no abnormal blood routine results, coagulation function or liver function. Blood tumor markers, including $\alpha$-fetoprotein (AFP), carcinoembryonic antigen (CEA), cancer antigens (CA)-19-9, $-72-4$ and -125 , were all within normal ranges $(<10,<3 \mathrm{ng} / \mathrm{ml}$, $37,<7$ and $<46 \mathrm{U} / \mathrm{ml}$, respectively) and antibodies (Ab) against hepatitis A virus (HAV) and hepatitis $\mathrm{C}$ virus (HCV) were negative. The blood tests for hepatitis B virus (HBV) showed positive results for $\mathrm{HBeAb}$ and $\mathrm{HBcAb}$ and negative results for $\mathrm{HBsAg}, \mathrm{HBsAb}$ and $\mathrm{HBeAg}$.

A contrast-enhanced CT scan was performed (Fig. 1), which was identical to the previous CT scan, in which the non-enhanced phase revealed two adjacent low-density lesions, with diameters of 3.5 and $4.5 \mathrm{~cm}$ on segments IV and VIII of the liver, respectively. In the arterial phase, the edges of the two lesions were enhanced, but the centers were not. In the portal venous phase, the enhancement at the edge was 


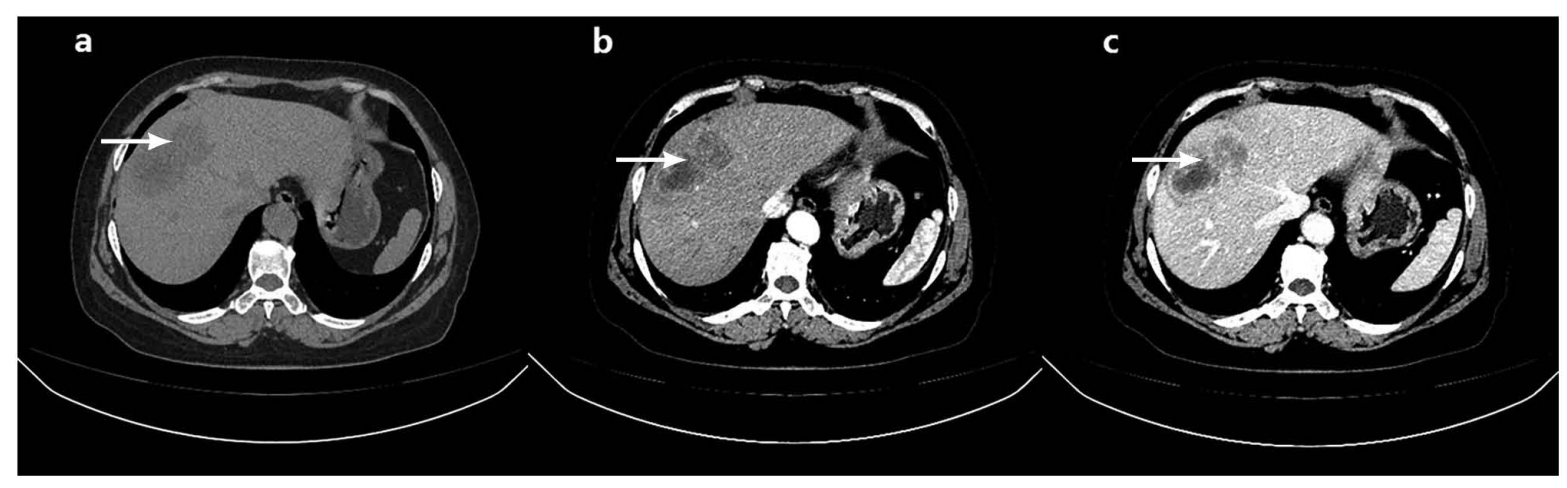

Figure 1. Contrast-enhanced computed tomography revealed multiple lesions on segments IV and VIII of the liver. (A) Non-enhanced phase. (B) Arterial phase. (C) Portal venous phase. The arrows indicate the multiple tumors.

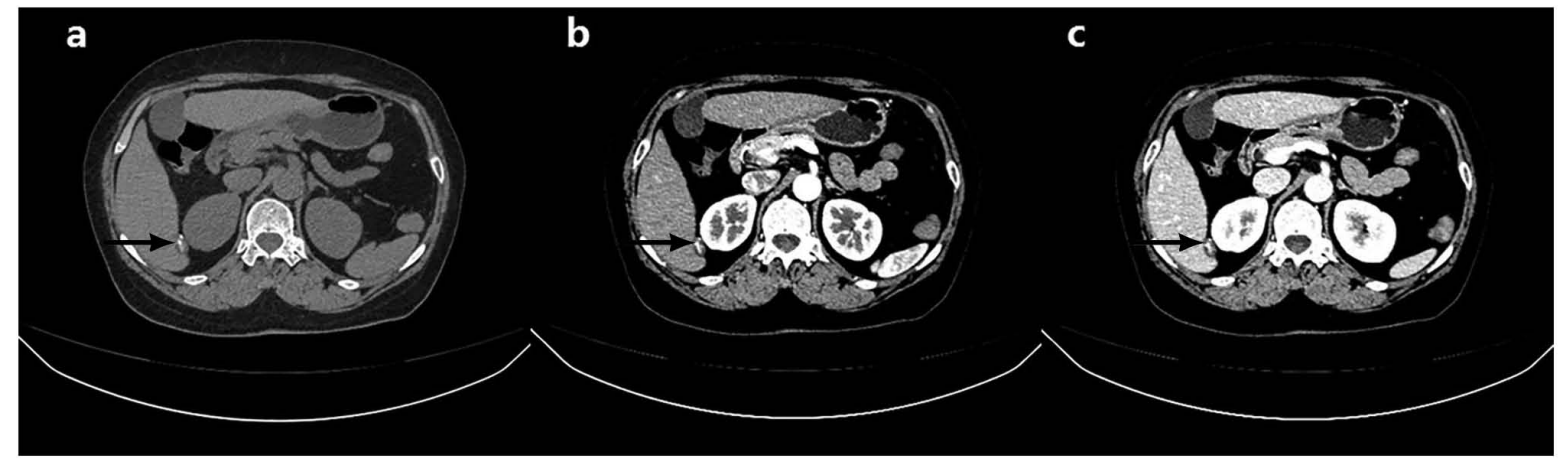

Figure 2. Contrast-enhanced computed tomography revealed a high-density nodule on the edge of segment VI of the liver. (A) Non-enhanced phase. (B) Arterial phase. (C) Portal venous phase. The arrows indicate the nodule.

markedly stronger, but the center remained unenhanced. Furthermore, a narrow low-density area could be observed between the lesions and normal liver tissue in the portal venous phase, showing a clear tumor boundary (Fig. 1). In addition, a high-density nodule was found on the edge of segment VI and was considered to be a calcification (Fig. 2).

As the diagnosis was not definitive based on the CT images alone, a PET/CT was subsequently performed, which revealed consistent results with the CT findings. The PET/CT scan revealed low-density lesions on segments IV and VII of the liver, with an increased glucose metabolism rate [early standard uptake value (SUV) max $_{\text {ax }}$ 4.4, delay $\mathrm{SUV}_{\max }$ 5.8] (Fig. 3). The nodule on segment VI revealed similar characteristics, and all lesions were considered to be malignant.

To rule out the possibility of metastatic liver tumors, gastroscopy and colonoscopy were also performed. No lesions were found during gastroscopy, although a polyp was found in the sigmoid colon using colonoscopy and was confirmed to be chronic mucosal inflammation using pathological examination by a pathologist from Department of Pathology at Hebei Medical University 4th Hospital. The following method was used for immunohistochemistry staining: Samples were fixed in $10 \%$ neutral buffered formalin at room temperature for $24 \mathrm{~h}$ and cut into $1 \times 1 \mathrm{~cm}$ sections. Slides were incubated with the primary antibodies (SMA, cat. no. kit-0006; 1:500; vimentin, cat. no. mab-0139; 1:500; CD34, cat. no. kit-0004; 1:100; CD31, cat. no. mab-0031; 1:500; desmin, cat. no. kit-0023; 1:2,000; HMB45, cat. no. mab-0098; 1:1,000; AE1/AE3, cat. no. kit-0009; 1:100; CK-7, cat. no. kit-0021; 1:1,000; Ki67, cat. no. kit-0005; 1:500; MART-1, cat. no. mab-0275; 1:1,000; glypican-3, cat. no. kit-0036; 1:100 and Hep-1, cat. no. mab- $0249 ; 1: 200$ ) overnight at $4{ }^{\circ} \mathrm{C}$, washed using $0.05 \%$ TBS-Tween-20 and then incubated with secondary antibodies and DAB using a kit (cat. no. TT-0801; prediluted) for $45 \mathrm{~min}$ (all Fuzhou Maixin Biotech Co., Ltd.). A light microscope was used for observation at x 200 magnification. Positive staining was determined by the pathologist, as there are no guidelines for IHC staining of myopericytoma.

Following the aforementioned examinations, the diagnosis indicated a primary malignant tumor of the liver and a partial hepatectomy was performed. The tumor on segments IV and VIII and the nodule on segment VI were completely excised. The tumor on segments IV and VIII had no clear capsule, and the section was tough and gray. The nodule on segment VI was hard, and calcifications were found on the tumor section. Pathological examination revealed blood vessels scattered throughout the tumor. Spindle myoid tumor cells with eosinophilic cytoplasm were concentrated around the blood vessels or were found to be arranged in spirals or bundles in some areas, as indicated by the arrow (Fig. 4A). Immunohistochemical results showed that the tumor was positive for smooth muscle actin (Fig. 4D), vimentin, CD-34 (Fig. 4B) and -31 (within the blood vessel) and negative for desmin, human melanoma black 45, cytokeratin (CK) AE1/AE3, CK7 (Fig. 4C), Ki67 (positive rate, 1\%), melanoma-associated antigen recognized by $\mathrm{T}$ cells 1 , glypican-3, 

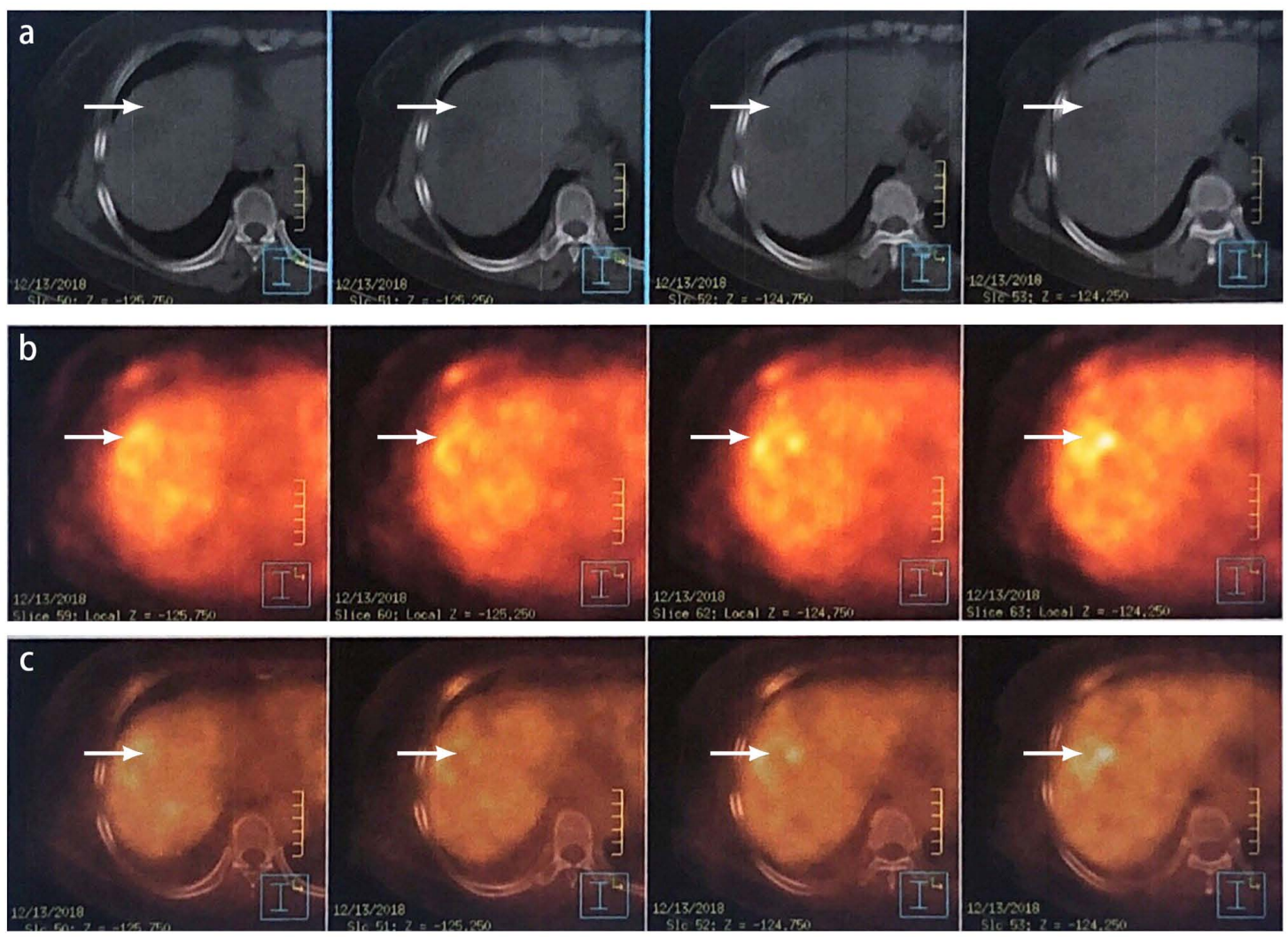

Figure 3. Positron emission tomography/computed tomography of the liver. Low-density lesions were found on segments IV and VII with an increased glucose metabolism rate. (A) Regular CT images. (B) Early stage (1 h following 18F-FDG injection) PET/CT images. (C) Delay stage (2 h following $18 \mathrm{~F}-\mathrm{FDG}$ injection) PET/CT images. Arrows indicate the lesions. PET, positron emission tomography; CT, computed tomography; 18F-FDG, fluorodeoxyglucose.

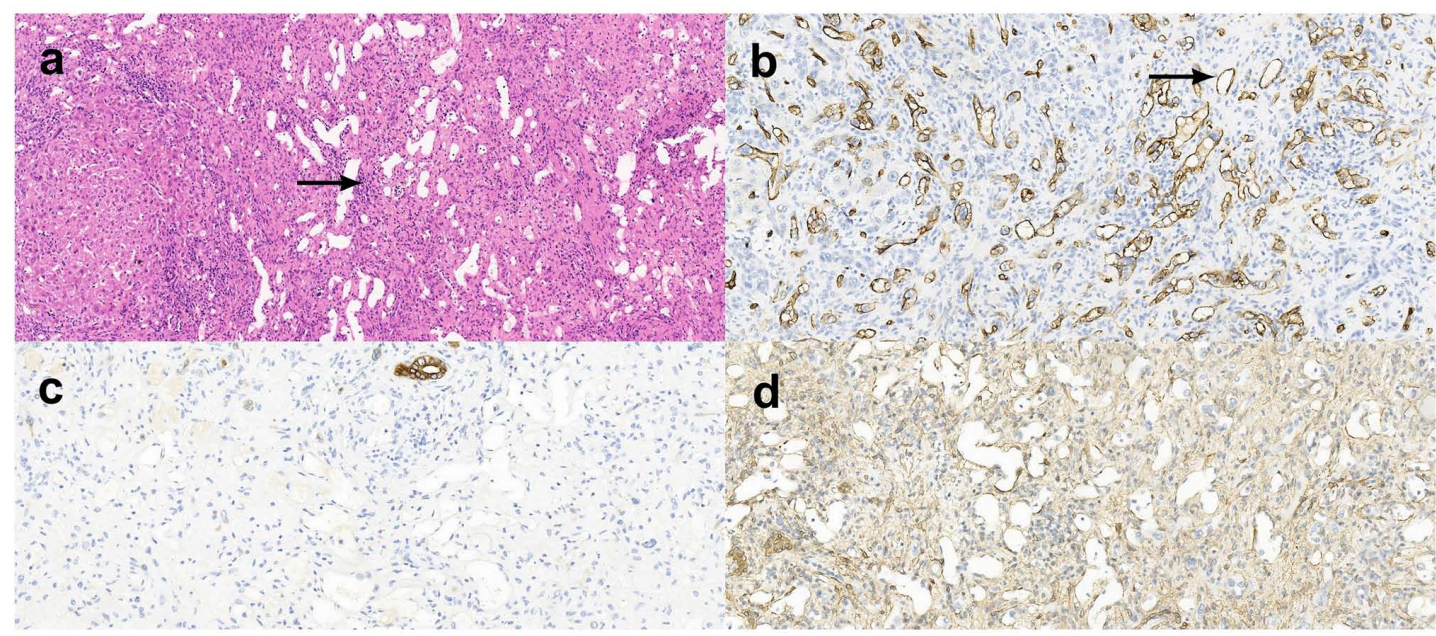

Figure 4. Pathological examination of lesions on segments IV, VI and VIII of the liver. (A) Hematoxylin and eosin staining (x100) showed spindle myoid tumor cells (as indicated by the arrow) growing around the blood vessels. (B) Positive CD34 staining in the blood vessels (as indicated by the arrow) (x200). (C) Negative cytokeratin 7 staining (x200). (D) Smooth muscle actin staining was positive in the majority of the tumor tissue (x200).

and Hep-1. All the lesions on segments IV, VI and VIII were confirmed to be myopericytomas from the aforementioned pathological examination. The patient was discharged 9 days following surgery, with no complications.

\section{Discussion}

Myopericytoma is a rare type of tumor that primarily occurs on the four limbs (6), and can occur in individuals of all ages but it is more commonly found in middle-aged men ( $>50$ years of age), with no symptoms $(3,6,9)$. Reports of myopericytoma occurring in the abdominal organs, particularly the liver, are extremely rare. Moreover, no imaging profiles of liver myopericytoma have been reported thus far. Therefore, the differential diagnosis of liver myopericytoma could be difficult prior to surgery, even with contrast-enhanced CT and PET/CT images. The imaging results from the present study can appear similar to those of several types of liver neoplasms, including 
hepatocellular carcinoma (HCC), hepatic hemangioma, intrahepatic cholangiocarcinoma and liver metastatic tumor. Thus, in the present report, the preoperative differential diagnosis of liver myopericytoma based on the imaging profile and laboratory examinations was difficult.

According to previous reports, the contrast-enhanced CT images of myopericytoma generally have a similar pattern. The tumors appear to be low-density lesions in the non-enhanced phase; however, the peripheral area of the tumor could be enhanced, showing rim-like enhancement in the arterial phase (11-16). Small tumors are displayed as full-tumor enhancement in the arterial phase (13). Enhancement, in which the contrast agent fills in centripetally, can be identified in some of the tumors over time, especially the large ones, leading to a heterogeneous enhancement in the center in the portal venous phase $(40-50 \mathrm{sec})$ and delayed phase $(100 \mathrm{sec}$ to $10 \mathrm{~min})(11,15,16)$. Calcifications were also observed in some tumors $(12,15,16)$. The contrast-enhanced CT image in the present case report was consistent with previous studies; however, the enhancement fill-in was not obvious in the portal venous phase. In this instance, the contrast-enhanced CT of the patient only included the portal venous phase but not the delayed phase, since the typical hallmark of liver neoplasms is similar between these two phases, therefore only the portal venous phase is shown in Fig. 2.

Both HCC and hepatic hemangioma have unique imaging and clinical features. Patients with $\mathrm{HCC}$ are known to have a background of liver cirrhosis caused by $\mathrm{HBV} / \mathrm{HCV}$ or alcohol intake (17). Increased AFP levels (>400 ng/ml) and the fast washout of contrast enhancement on $\mathrm{CT}$ are methods to diagnose HCC (17). For hepatic hemangioma, peripheral globular enhancement and a centripetal fill-in pattern on contrast-enhanced CT are unique diagnostic features (18). Liver myopericytoma lacks all of the aforementioned imaging and clinical characteristics, therefore the differential diagnosis is definitive.

In addition, the differential diagnosis of myopericytoma from intrahepatic cholangiocarcinoma and metastatic liver tumors, identified in the present study, can be confusing as these lesions all have peripheral rim-like enhancement on contrast-enhanced CT. However, unique imaging features still exist on each type of tumor.

For intrahepatic cholangiocarcinoma, peripheral rim-like enhancement can be observed in the arterial phase in the majority of cases, and a centripetal fill-in enhancement pattern can be observed in the portal phase and delayed phase in some cases $(19,20)$. In addition, intrahepatic bile duct dilatation proximal to the tumor and regional lymph node enlargement can occur in some cases $(19,20)$ and could be the primary difference between intrahepatic cholangiocarcinoma and liver myopericytoma. With respect to PET/CT, previous studies have found that intrahepatic cholangiocarcinoma had a ${ }^{18} \mathrm{~F}$-fluorodeoxyglucose median $\mathrm{SUV}_{\max }$ uptake ranging from 8.2 to 14.4 (21-23), which is higher compared with the $\mathrm{SUV}_{\max }$ of liver myopericytoma in the present case $\left(\mathrm{SUV}_{\max }\right.$, 5.8). Therefore, PET/CT has the potential to assist with the differential diagnosis; however, due to the lack of reports of myopericytoma PET/CT image profiles, the sensitivity and specificity requires further validation.

A complete ring of enhancement in the arterial phase of contrast-enhanced CT is the primary imaging feature of liver metastases, and centripetal fill-in can be observed in some cases during the portal venous phase or the delayed phase (24). As the imaging characteristics of liver metastases are very similar to those of myopericytoma, the differential diagnosis can be difficult if this is only based on the image of the tumor in the liver. Therefore, identifying the primary tumor is important for diagnosis and PET/CT could play an important role as the primary tumor can be observed at the same time. Gastroscopy and colonoscopy are also recommended to rule out metastases from gastric cancer and colorectal cancer.

In the present case report, other imaging techniques, such as MRI or contrast enhanced ultrasound examination in addition to contrast-enhanced CT and PET/CT were not performed. According to previously published clinical guidelines from the European Association for the Study of the Liver and the European Organization for Research and treatment of Cancer (17), only contrast-enhanced CT and magnetic resonance imaging (MRI) are recommended as non-invasive diagnostic methods for liver tumors (25). Due to the uncertainty of diagnosis following contrast-enhanced CT, PET/CT was used to identify the malignant nature of the tumor. However, additional imaging examinations, such as MRI or contrast-enhanced ultrasound, might provide beneficial imaging information for the differential diagnosis of this rare case.

Beyond imaging techniques, biomarkers are also important for the differential diagnosis of liver myopericytoma. At present, there are no peripheral blood biomarkers proven to be specific to myopericytoma. However, for intrahepatic cholangiocarcinoma, CEA and CA19-9 could significantly increase above the normal levels in the peripheral blood of the patients (20), and certain biomarker increases in the primary tumor could also be observed in patients with metastatic liver tumors, such as CA-19-9 and CEA (26).

In conclusion, to the best of our knowledge, this is the first case report of liver myopericytoma, with preoperative contrast-enhanced CT and PET/CT image profiles. The characteristic imaging results of liver myopericytoma includes peripheral rim-like enhancement in the arterial phase and a centripetal fill-in pattern in the portal venous phase and delayed phase, which is similar to intrahepatic cholangiocarcinoma and liver metastases. Intrahepatic bile duct dilatation, regional lymph node enlargement and extrahepatic primary tumor found by imaging techniques can be beneficial to exclude the diagnosis of liver myopericytoma prior to surgery. Blood biomarkers, including AFP, CEA, CA19-9, could also assist with the differential diagnosis between these tumors.

\section{Acknowledgements}

Not applicable.

\section{Funding}

No funding was received.

\section{Availability of data and materials}

All data generated or analyzed during this study are included in this published article. 


\section{Authors' contributions}

$\mathrm{XK}$ and SW performed the literature research and wrote the study. HG collected the data and image information. FL performed pathological diagnosis and described the images. All authors read and approved the final manuscript.

\section{Ethics approval and consent to participate}

Not applicable.

\section{Patient consent for publication}

The patient provided written informed consent for publication of this manuscript.

\section{Competing interests}

The authors declare that they have no competing interests.

\section{References}

1. Granter SR, Badizadegan K and Fletcher CD: Myofibromatosis in adults, glomangiopericytoma, and myopericytoma: A spectrum of tumors showing perivascular myoid differentiation. Am J Surg Pathol 22: 513-525, 1998.

2. Fletcher CDM, Unni KK and Mertens F (eds): World Health Organization Classification of Tumours Pathology and Genetics of Tumours of Soft Tissue and Bone. International Agency for Research on Cancer (IARC) Press, Lyon, 2002.

3. Dray MS, McCarthy SW, Palmer AA, Bonar SF, Stalley PD, Marjoniemi V, Millar E and Scolyer RA: Myopericytoma: A unifying term for a spectrum of tumours that show overlapping features with myofibroma. A review of 14 cases. J Clin Pathol 59: 67-73, 2006.

4. Numata I, Nakagawa S, Hasegawa S and Aiba S: A myopericytoma of the nose. Acta Derm Venereol 90: 192-193, 2010.

5. Zhao M, Williamson SR, Sun K, Zhu Y, Li C, Xia W, Qi H, Wang L, Linos K and Cheng L: Benign perivascular myoid cell tumor (myopericytoma) of the urinary tract: A report of 2 cases with an emphasis on differential diagnosis. Hum Pathol 45: $1115-1121,2014$

6. Mentzel T, Dei Tos AP, Sapi Z and Kutzner H: Myopericytoma of skin and soft tissues: Clinicopathologic and immunohistochemical study of 54 cases. Am J Surg Pathol 30: 104-113, 2006

7. Rousseau A, Kujas M, Van Effenterre R, Bocht AL, Carpentier A, Leroy JP and Poirier J: Primary intracranial myopericytoma: Report of three cases and review of the literature. Neuropathol Appl Neurobiol 31: 641-648, 2005.

8. Lau SK, Klein R, Jiang Z, Weiss LM and Chu PG: Myopericytoma of the kidney. Hum Pathol 41: 1500-1504, 2010.

9. Chen Z and Liang W: Myopericytoma occurrence in the liver and stomach space: Imaging performance. BMC Cancer 17: 143, 2017.

10. Mannan AASR, McGinty J and Theise N: Myopericytoma of the Liver. Am J Clin Pathol 146 (Suppl 1): S246, 2016.

11. Wu F, Sun J, Dong J, Wang X and Gao Q: Management of multicentric myopericytoma in the maxillofacial region: A case report. Case Rep Oncol 6: 350-355, 2013.
12. Prado-Calleros HM, Galarza-Lozano D, Arrieta-Gómez JR, Pombo-Nava A, Parraguirre-Martínez S and Gutiérrez CJ: Myopericytoma arising adjacent to the common carotid artery: Case report and systematic review of deep located neck myopericytomas. Head Neck 38: E2479-E2482, 2016.

13. Nagai T, Kamimura T, Itou K, Fujii M, Tsukino H, Mukai S, Akiyama Y, Kataoka $\mathrm{H}$ and Kamoto T: Myopericytoma in urinary bladder: A case report. J Med Case Rep 11: 46, 2017

14. Chu ZG, Yu JQ, Yang ZG, Zhu ZY and Yuan HM: Myopericytoma involving the parotid gland as depicted on multidetector CT. Korean J Radiol 10: 398-401, 2009.

15. Cao JH, Xu JP, Li YC, Lai J and Li Q: Pulmonary myopericytoma: A case report and review of the literatures. Chin Med J (Engl) 122: 755-757, 2009.

16. Zhang Z, Yu D, Shi H and Xie D: Renal myopericytoma: A case report with a literature review. Oncol Lett 7: 285-287, 2014

17. European Association for Study of Liver and European Organisation for Research and Treatment of Cancer: EASL-EORTC clinical practice guidelines: Management of hepatocellular carcinoma. Eur J Cancer 48: 599-641, 2012.

18. Quinn SF and Benjamin GG: Hepatic cavernous hemangiomas: Simple diagnostic sign with dynamic bolus CT. Radiology 182: 545-548, 1992.

19. Valls C, Gumà A, Puig I, Sanchez A, Andía E, Serrano T and Figueras J: Intrahepatic peripheral cholangiocarcinoma: CT evaluation. Abdom Imaging 25: 490-496, 2000.

20. Bridgewater J, Galle PR, Khan SA, Llovet JM, Park JW, Patel T, Pawlik TM and Gores GJ: Guidelines for the diagnosis and management of intrahepatic cholangiocarcinoma. J Hepatol 60: 1268-1289, 2014.

21. Albazaz R, Patel CN, Chowdhury FU and Scarsbrook AF: Clinical impact of FDG PET-CT on management decisions for patients with primary biliary tumours. Insights Imaging 4: 691-700, 2013.

22. Lee SW, Kim HJ, Park JH, Park D I, Cho YK, Sohn CI, Jeon WK and Kim BI: Clinical usefulness of 18F-FDG PET-CT for patients with gallbladder cancer and cholangiocarcinoma. J Gastroenterol 45: 560-566, 2010

23. Petrowsky H, Wildbrett P, Husarik DB, Hany TF, Tam S, Jochum W and Clavien PA: Impact of integrated positron emission tomography and computed tomography on staging and management of gallbladder cancer and cholangiocarcinoma. J Hepatol 45: 43-50, 2006.

24. Sica GT, Ji H and Ros PR: CT and MR imaging of hepatic metastases. Am J Roentgenol 174: 691-698, 2000.

25. Galle PR, Forner A, Llovet JM, Mazzaferro V, Piscaglia F, Raoul JL, Schirmacher P and Vilgrain V: EASL Clinical Practice Guidelines: Management of hepatocellular carcinoma. J Hepatol 69: 182-236, 2018.

26. Locker GY, Hamilton S, Harris J, Jessup JM, Kemeny N, Macdonald JS, Somerfield MR, Hayes DF and Bast RC Jr; ASCO: ASCO 2006 update of recommendations for the use of tumor markers in gastrointestinal cancer. J Clin Oncol 24: 5313-5327, 2006. 